\title{
PERDA DE CARGA LOCALIZADA EM EMISSORES NÃO COAXIAIS INTEGRADOS A TUBOS DE POLIETILENO
}

\author{
OSVALDO RETTORE NETO ${ }^{1}$, JOSÉ A. FRIZZONE ${ }^{2}$, JARBAS H. MIRANDA ${ }^{3}$, \\ TARLEI A. BOTREL ${ }^{4}$
}

\begin{abstract}
RESUMO: Este trabalho apresenta os resultados de experimento conduzido para avaliar as perdas de carga localizadas em gotejados não coaxiais integrados a tubos de polietileno. As perdas de carga para diferentes vazões foram determinadas em quatro modelos de tubos-gotejadores, com dez repetições. Para cada vazão, a perda de carga localizada foi calculada pela diferença entre a perda de carga no tubo com emissor e a perda de carga contínua no tubo uniforme estimada pela equação de Darcy-Weisbach com o fator de atrito $(f)$ previamente determinado. Aproximações matemáticas foram sugeridas para calcular $h f e$ com base no coeficiente de carga cinética $(K)$ e em um valor constante de comprimento equivalente $(L e)$. Para os modelos de tubos-gotejadores estudados, a perda de carga localizada, expressa como percentagem da perda de carga total, aumentou com o aumento da razão de obstrução, variando de $24,5 \%$ a $50,8 \%$ para $A g / A t=0,221$ e 0,429 , respectivamente. A maior diferença percentual encontrada entre a perda de carga total calculada pelo método iterativo passo a passo e pelo uso de " $L e$ " constante, calculado com a vazão de entrada na lateral, foi 5,5\% para o gotejador não autocompensante. Para os gotejadores autocompensantes, as diferenças foram inferiores a $1,7 \%$.
\end{abstract}

PALAVRAS-CHAVE: coeficiente de carga cinética, comprimento equivalente, irrigação por gotejamento, fator de atrito.

\section{LOCAL HEAD LOSS FOR UNCOAXIAL DRIPPERS INSERTED IN POL YETHYLENE PIPES}

\begin{abstract}
In this paper, the results of an experimental investigation on local head losses from uncoaxial in-line emitters inserted into the pipe line are reported. Local losses corresponding to four different pipe-emitter combinations, with ten replications, were measured for a range of discharge values. For each discharge, the amount of local losses was calculated as the difference between the total measured head losses and the corresponding friction losses evaluated by the Darcy-Weisbach equation. Mathematical approaches were tested to calculate hfe based on the kinetic head coefficient $(K)$ and constant equivalent length $(L e)$. For the drip-line models examined, the amount of local losses, expressed as a percentage of the total losses, was found to grow with an increase in the obstruction degree. The percentage increased to $24.5 \%$ and $50.8 \%$, respectively, for $A g / A t=$ 0.221 and 0.429 . The results of this experimental investigation indicated the practical validity of using a constant equivalent length $(L e)$ to estimate the local head losses in a drip lateral line. The percentage variation between total head loss calculated by a step-by-step procedure and the Le approximation method was $5.5 \%$ for non-compensating pressure emitters and less than $1.7 \%$ for the pressure compensating emitters.
\end{abstract}

KEYWORDS: kinetic head coefficient, equivalent length, drip irrigation, friction factor.

\footnotetext{
${ }^{1}$ Eng ${ }^{\circ}$ Agrícola, M.S. em Irrigação e Drenagem, Departamento de Engenharia Rural, ESALQ/USP - Piracicaba - SP, Fone: (19) 3447-8550, orettore@esalq.usp.br

${ }^{2}$ Eng $^{\circ}$ Agrônomo, Prof. Titular, Departamento de Engenharia Rural, ESALQ/USP, Piracicaba - SP.

${ }^{3}$ Eng $^{\mathrm{O}}$ Agrônomo, Prof. Associado, Departamento de Ciências Exatas, ESALQ/USP, Piracicaba - SP.

${ }^{4}$ Eng ${ }^{0}$ Agrícola, Prof. Associado, Departamento de Engenharia Rural, ESALQ/USP, Piracicaba - SP.

Recebido pelo Conselho Editorial em: 9-6-2008

Aprovado pelo Conselho Editorial em: 5-3-2009
}

Eng. Agríc., Jaboticabal, v/29, n.1, p.28-39, jan./mar. 2009 


\section{INTRODUÇÃO}

O projeto hidráulico dos sistemas por gotejamento deve ser realizado para atingir alta uniformidade de distribuição de água. Entre outras causas, a vazão dos gotejadores ao longo da lateral é afetada pela perda de carga total na tubulação e pelo desnível do terreno. A perda de carga total é um dos fatores que afeta fortemente a pressão disponível nos gotejadores e, em consequência, a distribuição da vazão é significativamente afetada quando são utilizados gotejadores não autocompensantes.

A perda de carga total é estimada pela soma da perda de carga contínua, ao longo das seções uniformes de tubo entre os gotejadores consecutivos, com a perda de carga localizada nos gotejadores "in-line" ou nas conexões dos gotejadores "on-line". Essas perdas de carga possuem ordens de grandeza semelhantes (HOWELL \& BARINAS, 1980; AL-AMOUD, 1995). Enquanto a determinação das perdas de carga contínuas já é bem definida pelos modelos hidráulicos existentes, os projetistas geralmente falham na estimativa das perdas localizadas por serem mais complexas.

A perda de carga em escoamentos turbulentos permanentes e uniformes de fluidos reais, através de tubos de seção cilíndrica, pode ser calculada por diferentes equações. A contribuição mais importante é expressa pela equação de Darcy-Weisbach, cuja expressão é dada pela eq.(1) (ROMEO et al. 2002):

$$
\mathrm{h}_{\mathrm{f}}=\mathrm{f} \frac{\mathrm{L}}{\mathrm{D}} \frac{\mathrm{V}^{2}}{2 \mathrm{~g}}
$$

em que,

$\mathrm{h}_{\mathrm{f}}$ - perda de carga contínua na tubulação, $\mathrm{m}$;

L - comprimento do tubo, $\mathrm{m}$;

D - diâmetro interno do tubo, m;

$\mathrm{V}$ - velocidade média do escoamento, $\mathrm{m} \mathrm{s}^{-1}$;

$\mathrm{g}$ - aceleração da gravidade, $\mathrm{m} \mathrm{s}^{-2}$, e

$\mathrm{f}$ - fator de atrito, dependente do número de Reynolds $(\mathrm{R})$ e da altura das asperezas $(\varepsilon, \mathrm{em} \mathrm{m})$ nas paredes do tubo.

Na equação de Darcy-Weisbach, a estimativa do fator de atrito $(f)$ é essencial para o cálculo da perda de carga contínua. Para o escoamento laminar $(R<2.000)$, o cálculo de $f$ é feito pela equação de Hagen-Poiseuille $(f=64 / R)$, sendo apenas uma função do número de Reynoldos $(R)$, que depende exclusivamente das propriedades do fluido, do diâmetro do tubo e da velocidade do escoamento.

No escoamento permanente turbulento e uniforme em tubos, a estimativa do fator de atrito é mais complexa, pois $f$ é uma função da rugosidade relativa das paredes do tubo $(\varepsilon / D)$ e do número de Reynolds. Para $4.000 \leq R<10^{8}$ e $0 \leq \varepsilon / D 0,05$, a equação de Colebrook-White é a mais utilizada para calcular $f$ (YOO \& SINGH, 2005), relacionando o fator de atrito com a rugosidade relativa e com o número de Reynolds, sendo válida também para o caso limite de tubos lisos $(\varepsilon=0)$. Para o regime de transição $(2.000 \leq R<4.000)$, as características do escoamento são indeterminadas.

No escoamento turbulento uniforme em tubos lisos, o tamanho das asperezas não influi sobre a turbulência do escoamento, e o coeficiente $f$ independe da rugosidade das paredes internas do tubo, podendo ser calculado em função do número de Reynolds. Para $4000 \leq R \leq 10^{5}$, o cálculo do fator de atrito pode ser feito por uma equação simples na forma proposta por Blasius (BERNUTH, 1990), cuja equação é uma função somente do número de Reynolds, sendo apresentada pela eq.(2):

$$
\mathrm{f}=\frac{\mathrm{c}}{\mathrm{R}^{\mathrm{m}}}
$$


Ao propor a eq.(2) para calcular $f$, Blasius determinou $m$ como uma constante de valor igual a 0,25 , enquanto o coeficiente $(c)$ seria outra constante de valor igual a 0,316. Para BERNUTH (1990), a inserção do fator de atrito de Blasius na equação de Darcy-Weisbach resulta em uma equação combinada com as seguintes vantagens: (i) é teoricamente perfeita e dimensionalmente homogênea, pois tanto a equação de Darcy-Weisbach quanto a de Blasius têm bases teóricas; (ii) tem bom grau de exatidão para tubos lisos quando $4.000 \leq R \leq 10^{5}$, sendo que o número de Reynolds limite não é restritivo para sistemas de irrigação que usam tubos com diâmetros inferiores a $80 \mathrm{~mm}$, e (iii) pode ser facilmente corrigida para variações na viscosidade da água.

Alternativas empíricas para determinar $f$ por ensaios de laboratório satisfazem à expectativa de obter resultados aceitáveis, como se observa nos trabalhos de BERNUTH (1990), que apresentam bons resultados usando equações do tipo potência, semelhante à de Blasius. BAGARELLO et al. (1995) mostraram que os coeficientes propostos por Blasius superestimam os valores de $f$ para os atuais tubos de polietileno. Esses autores analisaram tubos de polietileno de diâmetros nominais de 16;20 e $25 \mathrm{~mm}$, variando o número de Reynolds pela mudança da viscosidade do fluido $(3.037<R<31.373)$ ao se alterar a temperatura, obtendo $c=0,302$ para $m=0,25$.

Para $2.000 \leq R<4.000$, o regime de escoamento é indeterminado, e a aplicação da eq.(2) com os coeficientes de Blasius pode superestimar o fator $f$ em mais de cinco vezes (BERNUTH \& WILSON, 1989), embora a perda de carga nesse regime de escoamento seja muito pequena. Para este caso, o fator $f$ pode ser aproximado pela eq.(3), conforme recomenda SILVERBERG \& MANADILI (1997):

$$
\mathrm{f}=2,8210^{-7} \mathrm{R}^{1,52}
$$

Os gotejadores integrados nas paredes internas dos tubos reduzem a seção de escoamento no ponto de inserção e determinam perda de carga localizada nesse ponto. Essa perda de carga deve-se à resistência à movimentação da corrente fluida oferecida pelo elemento obstrutor no interior do tubo, sendo expressa na forma clássica como uma fração $K$ da carga cinética [eq.(4)]:

$$
\mathrm{h}_{\mathrm{fe}}=\mathrm{K} \frac{\mathrm{V}^{2}}{2 \mathrm{~g}}
$$

em que,

$\mathrm{h}_{\mathrm{fe}}$ - perda de carga localizada, $\mathrm{m}$;

$\mathrm{V}$ - velocidade média de aproximação da corrente fluida, $\mathrm{m} \mathrm{s}^{-1}$, e

$\mathrm{K}$ - coeficiente de carga cinética ou de resistência de perfil.

Aumentando-se a velocidade de escoamento, maiores serão as perdas localizadas, uma vez que a turbulência do fluido na passagem entre o elemento obstrutor e a parede do tubo tende a aumentar.

O coeficiente $K$ sintetiza os efeitos da contração e do alargamento brusco da seção de escoamento sobre a perda de carga, podendo ser expresso em função de um índice de obstrução $\left(K=\varepsilon I O^{\eta}\right)$, desde que $K$ possa ser considerado independente de $R$ (BAGARELLO et al., 1997). O índice de obstrução $(I O)$ depende da razão de obstrução $(r): I O=(1-r)^{2} / r^{2}$, sendo $r$ a razão entre a área livre para escoamento no ponto de inserção do gotejador $\left(A_{r}\right)$ e a área da seção transversal do tubo $\left(A_{t}\right)$.

O objetivo deste trabalho foi analisar a perda de carga localizada decorrente de gotejadores não coaxiais integrados a tubos de polietileno e propor modelos empíricos, a partir das equações de Blasius, de Darcy-Weisbch e da teoria proposta por BAGARELO et al. (1997), para a estimativa das perdas de carga localizada e total, como suporte para o dimensionamento de sistemas de irrigação por gotejamento. 


\section{MATERIAL E MÉTODOS}

O experimento foi desenvolvido no Laboratório de Irrigação, do Departamento de Engenharia Rural - ESALQ/USP, Piracicaba - SP. Construiu-se uma bancada de ensaio de perda de carga para o controle, o monitoramento e a aquisição dos dados necessários ao desenvolvimento do trabalho (Figura 1).

Utilizou-se de um reservatório e uma motobomba ligada a um inversor de frequência com a finalidade de manter a rotação do motor constante durante os ensaios, evitando alterações de vazão provocadas pela variação de voltagem na rede de alimentação. No recalque, foi instalado um filtro de disco de 1". Logo após, foi conectada uma válvula de gaveta para controlar a pressão na entrada da linha, que foi monitorada por manômetro digital com faixa de trabalho de 0 a $1.500 \mathrm{kPa}$, com precisão de $1 \mathrm{kPa}$. Durante os ensaios, a pressão foi mantida entre 145 e $155 \mathrm{kPa}$ a fim de minimizar a alteração do diâmetro dos tubos, cujo efeito na perda de carga foi relatado por VILELA et al. (2003). A vazão foi medida por medidor magnético indutivo instalado no final da tubulação, com $1 \%$ de precisão e, após a passagem pelo medidor, a água retornava ao reservatório.

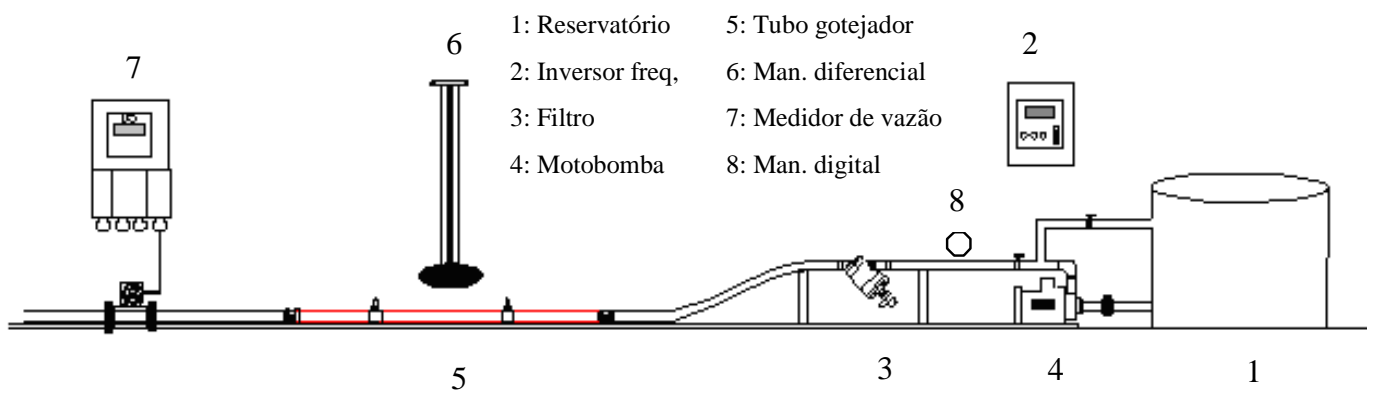

FIGURA 1. Esquema da instalação experimental para a determinação da perda de carga. Experimental installation layout for head loss.

A determinação da perda de carga distribuída foi realizada por meio de manômetro diferencial em "U", contendo líquido manométrico de densidade 1,5 e divisão de escala de $1,0 \mathrm{~mm}$. A diferença de pressão foi determinada em segmentos de tubo de 1,0 m de comprimento e as conexões do manômetro diferencial foram feitas a $0,25 \mathrm{~m}$ das extremidades desse segmento (Figura 1). $\mathrm{O}$ diâmetro interno médio dos tubos foi de $15,53 \mathrm{~mm}$, medido por projeto óptico de perfil, Starret, modelo HB400, acoplado a um microcomputador. Na Tabela 1, apresentam-se o valor médio do diâmetro do tubo, a espessura da parede, o desvio-padrão e o coeficiente de variação.

Os ensaios foram realizados de forma a medir a diferença de pressão entre as extremidades, em função da vazão que escoava através do tubo. A diferença de pressão para o segmento de tubo em nível representou a perda de carga em $0,5 \mathrm{~m}$ de comprimento. $\mathrm{O}$ número de vazões utilizadas foi, no mínimo, 19, e no máximo, 21, para cada tubo, com dez repetições. Durante os ensaios, monitorou-se a temperatura da água, que variou de 20,5 a $24,5^{\circ} \mathrm{C}$, sendo utilizada para corrigir a viscosidade cinemática da água nos cálculos da perda de carga.

TABELA 1. Características do tubo utilizado no ensaio. Tube characteristics used in the experiment.

\begin{tabular}{lccc}
\hline Parâmetros & Média $(\mathrm{mm})$ & Desvio-Padrão $(\mathrm{mm})$ & Coeficiente de Variação $(\%)$ \\
\hline Diâmetro interno & 15,53 & 0,134 & 0,86 \\
Espessura da parede & 1,07 & 0,061 & 5,70 \\
\hline
\end{tabular}

Os valores experimentais da perda de carga foram utilizados para calcular o fator de atrito $(f)$ pela eq.(1), conhecidos os valores de $V^{2} / 2 g$ e $D$ para $L=1$. O valor de $c$ da eq.(2) foi determinado para $m=0,25$, por meio de regressão linear entre $f$, calculado com as perdas de carga observadas, e $R^{-0,25}$. 
Para a determinação da perda de carga localizada, utilizou-se de quatro modelos de gotejadores não coaxiais integrados à parede do tubo, com as características hidráulicas e geométricas apresentadas na Tabela 2. Para cada modelo, foram analisadas dez amostras de tubo-gotejador e, devido aos diferentes espaçamentos entre emissores, analisaram-se segmentos de tubos de três comprimentos diferentes: 1,4 m para o Tiran, 1,5 m para o Uniram e o Drip Net PC, e 2,0 m para o Twin Plus. Os emissores foram vedados para manter constante a vazão de entrada e de saída no tubo durante os ensaios.

Os microtubos do manômetro diferencial foram conectados ao tubo gotejador por meio de conexão de silicone, a $0,5 \mathrm{~m}$ da extremidade do emissor, de ambos os lados. Determinou-se a perda de carga entre os dois pontos para 15 valores de vazão, sendo a vazão média inicial $0,3421 \mathrm{~m}^{3} \mathrm{~h}^{-1} \mathrm{e}$ final $1,2014 \mathrm{~m}^{3} \mathrm{~h}^{-1}$, que correspondeu à velocidade máxima de $2 \mathrm{~m} \mathrm{~s}^{-1}$. A temperatura da água durante o ensaio foi registrada para uso posterior no equacionamento matemático.

Para a tubulação em nível, contendo um emissor vedado, considerou-se a perda de carga no segmento de tubo como sendo a diferença de pressão entre as duas extremidades de medição. A perda de carga observada no tubo com emissor foi analisada em função da vazão, utilizando-se de modelo tipo potência, na forma da eq.(5):

$$
\mathrm{J}^{\prime}=\mathrm{a} \mathrm{Q} \mathrm{Q}^{\mathrm{b}}
$$

em que,

$\mathrm{J}^{\prime}$ - perda de carga unitária, $\mathrm{m} \mathrm{m}^{-1}$;

$\mathrm{Q}$ - vazão, $\mathrm{m}^{3} \mathrm{~h}^{-1}$, e

$\mathrm{a}$ e b - constantes.

TABELA 2. Características geométricas e hidráulicas dos conjuntos tubos-gotejadores. Geometric and hydraulic characteristics of set drip pipe.

\begin{tabular}{|c|c|c|c|c|}
\hline \multirow{2}{*}{ Características } & \multicolumn{4}{|c|}{ Gotejador } \\
\hline & Twin Plus $\ddagger$ & Tiran $\dagger$ & Uniram $\ddagger$ & Drip Net $\neq$ \\
\hline Diâmetro nominal do tubo & 17,5 & $17 \mathrm{D}$ & 17D & 17 \\
\hline Diâmetro interno do tubo (mm) & 15,5 & 14,4 & 14,4 & 15,2 \\
\hline Pressão de operação do gotejador (kPa) & $100-350$ & $50-300$ & $50-350$ & $40-250$ \\
\hline Vazão nominal do gotejador $\left(\mathrm{L} \mathrm{h}^{-1}\right)$ & 1,8 & 2,0 & 2,3 & 1,6 \\
\hline Espaçamento entre gotejadores (m) & 1,00 & 0,70 & 0,75 & 0,75 \\
\hline Área da seção interna do tubo, $A_{t}\left(\mathrm{~mm}^{2}\right)$ & 188,7 & 162,9 & 162,9 & 181,5 \\
\hline - Coeficiente de variação (\%) & 1,7 & 1,4 & 0,9 & 1,5 \\
\hline Área transversal do gotejador, $A_{g}\left(\mathrm{~mm}^{2}\right)$ & 57,1 & 35,9 & 69,9 & 53,7 \\
\hline - Coeficiente de variação (\%) & 1,0 & 1,4 & 3,3 & 1,1 \\
\hline Área livre para escoamento $\left(A_{r}\right)\left(\mathrm{mm}^{2}\right)$ & 131,6 & 127,0 & 93,0 & 127,8 \\
\hline Perímetro molhado (mm) & 58,7 & 49,1 & 51,8 & 52,8 \\
\hline - Coeficiente de variação (\%) & 0,4 & 0,3 & 1,1 & 0,9 \\
\hline Comprimento do gotejador (mm) & 36,1 & 72,0 & 44,6 & 21,5 \\
\hline - Coeficiente de variação (\%) & 0,3 & 0,1 & 0,3 & 0,8 \\
\hline
\end{tabular}

$\ddagger$ Autocompensante; $\dagger$ Não autocompensante.

A perda de carga provocada pelo emissor $\left(h_{f e}, \mathrm{em} \mathrm{m}\right)$ foi determinada em função da diferença entre a perda de carga unitária no tubo com um gotejador $\left(J^{\prime}, \mathrm{em} \mathrm{m} \mathrm{m}^{-1}\right)$ e a perda de carga unitária no tubo sem gotejador $\left(J\right.$, em $\left.\mathrm{m} \mathrm{m}^{-1}\right)$, calculada pela equação de Darcy-Weisbach com $f$ determinado para o tubo sem emissor, do comprimento do segmento de tubo ( $L$, em $\mathrm{m}$ ) e do número de emissores $(N)$ no segmento de comprimento $L$ [eq.(6)]:

$$
\mathrm{h}_{\mathrm{fe}}=\frac{\mathrm{J}-\mathrm{J}}{\mathrm{N}} \mathrm{L}
$$


A perda de carga no gotejador $\left(h_{f e}, \mathrm{em} \mathrm{m}\right)$ foi ajustada em função da vazão $\left(Q, \mathrm{em} \mathrm{m}^{3} \mathrm{~s}^{-1}\right)$, utilizando-se de modelo potencial [eq.(7)]:

$$
\mathrm{h}_{\mathrm{fe}}=\alpha \mathrm{Q}^{\mathrm{k}}
$$

Para a determinação da perda de carga localizada no emissor, em termos de comprimento equivalente de tubulação $\left(L_{e}\right)$, utilizou-se das equações de Darcy-Weisbach [eq.(1)] e da perda de carga localizada [eq.(4)], conforme REDDY (2003). Rearranjando os termos dessas equações, $L_{e}(\mathrm{~m})$ pode ser expresso pela eq.(8):

$$
\mathrm{L}_{\mathrm{e}}=\frac{\mathrm{K} \mathrm{D}}{\mathrm{f}}=\frac{2 \mathrm{~g} \mathrm{~h}_{\mathrm{fe}} \mathrm{D}}{\mathrm{f} \mathrm{V}^{2}}
$$

Desenvolvendo-se a eq.(8) para comprimento equivalente e utilizando a eq.(2) para escoamento turbulento permanente em tubos lisos, com $4.000<R<10^{5}$, tem-se:

$$
L_{e}=\frac{2 g_{f e} D}{c\left(\frac{4 Q v^{-1}}{\pi D}\right)^{-0,25}\left(\frac{4 Q}{\pi D^{2}}\right)^{2}}
$$

\section{RESULTADOS E DISCUSSÃO}

$\mathrm{Na}$ Figura 2(a), apresenta-se a perda de carga unitária $\left(\mathrm{m} \mathrm{m}^{-1}\right)$ para os tubos estudados, em função da vazão. Verifica-se que há relação potencial entre $Q$ e $h_{f}$, com $\mathrm{R}^{2}=0,9938$. Isso significa que $99,38 \%$ das variações da perda de carga podem ser explicadas pela variação da vazão. O expoente da vazão foi 1,733, caracterizando-se escoamento turbulento. Esse expoente difere de $0,97 \%$ do expoente da equação de perda de carga de Darcy-Weisbach com $f$ calculado pela equação de Blasius $(1,75)$.

Na Figura 2(b), apresenta-se a relação entre $f$ e $R^{-0,25}$, com $\mathrm{R}^{2}=0,8217$, para $8.244 \leq R$ $\leq 35.127$. A determinação dos coeficientes do modelo de Blasius também foi objeto de estudo de BAGARELLO et al. (1995), utilizando tubos de 16; 20 e $25 \mathrm{~mm}$ de diâmetro, e número de Reynolds variando entre 3.037 e 31.373 . Mediante análise semiteórica, esses autores obtiveram $c=0,302$ para $m=0,25$, enquanto ALVES \& PORTO (2002) encontraram $c=0,295$ para tubos de polietileno, ambos próximos do valor $c=0,296$ encontrado neste estudo. Comparando-se o coeficiente $c=0,302$ com o valor $c=0,296$, verifica-se diferença de apenas 2,0\%. Maior diferença $(6,3 \%)$ é encontrada ao se comparar com o valor tradicional da equação de Blasius $(0,316)$.

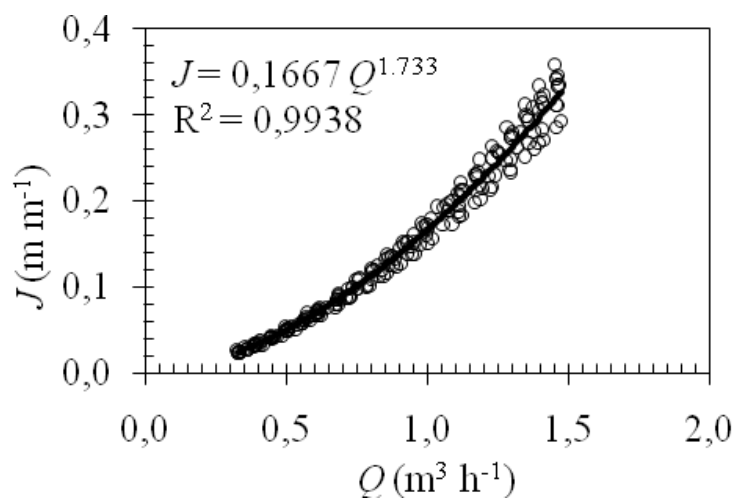

(a)

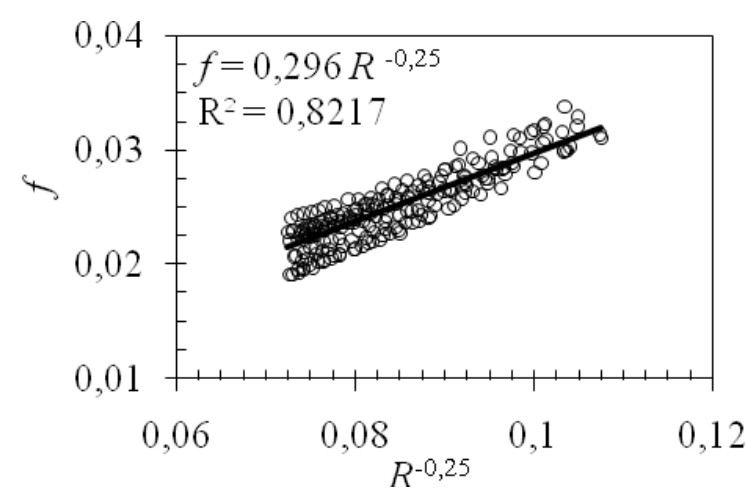

(b)

FIGURA 2. Perda de carga contínua para o tubo de polietileno (a); relação entre o fator de atrito $(f)$ e $R^{-0,25}$ obtido pelo ajuste dos dados experimentais com $m=0,25$ (b). Friction loss along the polyethylene pipe (a); friction factor $(f)$ and $R^{-0.25}$ relationship obtained by experimental data with $m=0.25$ (b). 
Na Figura 3, são apresentadas as relações entre a perda de carga localizada $\left(h_{f e}\right)$ e a carga cinética $\left(V^{2} / 2 g\right)$ para os gotejadores estudados. Verifica-se que o gotejador Tiran apresentou o menor coeficiente de carga cinética $(K=0,3378)$, e o Uniram, o maior coeficiente $(K=1,2719)$, correspondendo, respectivamente, à menor $(22,1 \%)$ e à maior $(42,9 \%)$ percentagem de obstrução da seção de escoamento. Para o gotejador Drip Net, obteve-se a maior dispersão dos valores de perda de carga nas amostras analisadas, o que pode ser justificado pelos frequentes desalinhamentos longitudinais observados na fixação do gotejador com a parede interna do tubo.

Verificou-se relação potencial significativa entre $Q$ e $h_{f e}$, para todos os modelos de gotejadores, pelo teste $\mathrm{F}$, a $1 \%$ de probabilidade. Na Tabela 3, apresentam-se os coeficientes das equações de perda de carga localizada $\left(h_{f e}\right)$, para cada modelo de gotejador, e os respectivos coeficientes de determinação para o ajuste. Também são apresentados os valores máximos e mínimos observados dos coeficientes $K$, nos quais se observa que a maior variação foi para o gotejador Drip Net, e a menor, para o Twin Plus, conferindo menor incerteza na estimativa da perda de carga localizada para esse emissor.
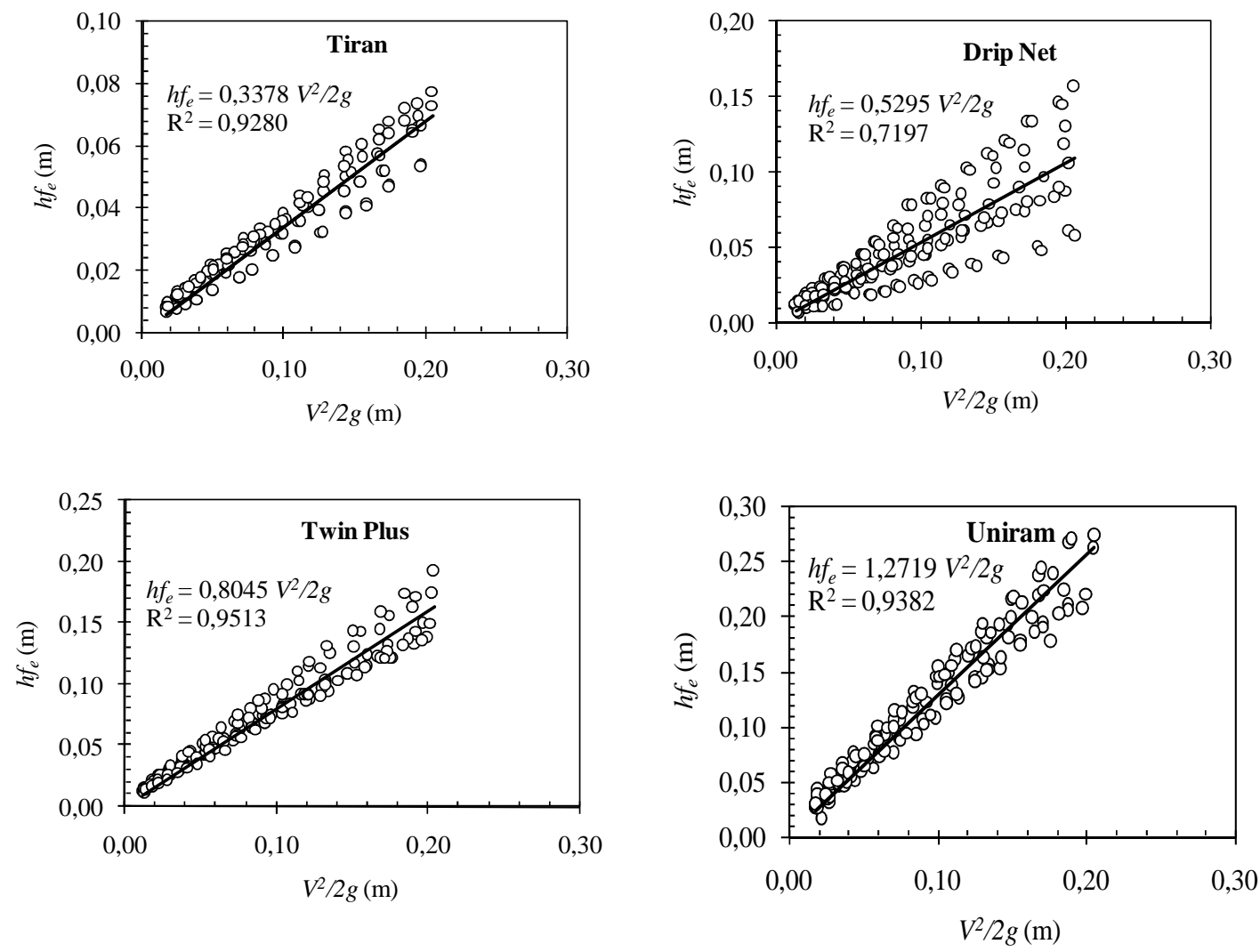

FIGURA 3. Perda de carga no emissor $\left(h_{f e}, \mathrm{~m}\right)$ em função da carga cinética $\left(V^{2} / 2 g, \mathrm{~m}\right)$. Local head loss at the emitter $\left(h f_{e}, \mathrm{~m}\right)$ in relation to kinetic load $\left(V^{2} / 2 g, \mathrm{~m}\right)$. 
TABELA 3. Coeficientes do modelo de perda de carga localizada no emissor $\left(h_{f e}\right.$, em m), em função da vazão $\left(Q\right.$, em $\left.\mathrm{m}^{3} \mathrm{~s}^{-1}\right)$, e intervalo de variação do coeficiente de carga cinética $(K)$. Head loss model coefficients in emitter $\left(\boldsymbol{h}_{f e}, \mathbf{m}\right)$, in function of flow $\left(Q, \mathrm{~m}^{3} \mathrm{~s}^{-1}\right)$ and variation interval of kinetic load coefficient $(K)$.

\begin{tabular}{lcccc}
\hline \multirow{2}{*}{ Coeficientes } & \multicolumn{4}{c}{ Gotejador } \\
\cline { 2 - 5 } & Tiran & Drip Net & Twin Plus & Uniram \\
\hline$\alpha$ & $9,6677510^{4}$ & $12,9793210^{4}$ & $16,0293810^{4}$ & $41,3567110^{4}$ \\
$k$ & 1,7412 & 1,7586 & 1,7601 & 1,7755 \\
$\mathrm{R}^{2}$ & 0,9448 & 0,7984 & 0,9791 & 0,9528 \\
$K$ mínimo & 0,2636 & 0,2676 & 0,7249 & 1,0674 \\
$K$ máximo & 0,3925 & 0,7668 & 0,9681 & 1,4845 \\
$R$ & $7.480-31.227$ & $7.748-32.372$ & $7.588-32.597$ & $8.316-29.897$ \\
\hline
\end{tabular}

O coeficiente $K$ depende do número de Reynolds e das características geométricas do elemento obstrutor. Na Figura 4, observa-se que os valores de $K$, para cada gotejador estudado, variam muito pouco com $R$ a partir de, aproximadamente, $R>10.000$. Para valores de $R<10.000, K$ aumenta com a redução de $R$, o que também foi observado por BAGARELLO et al. (1997) e PROVENZANO \& PUMO (2004). Como resultado, pode-se considerar que o efeito das forças viscosas é desprezível para $R>10.000$, e $K$ passa a depender, em grande parte, da forma e do tamanho do elemento obstrutor, e o valor médio de $K$ pode ser previsto a partir de um índice de obstrução.

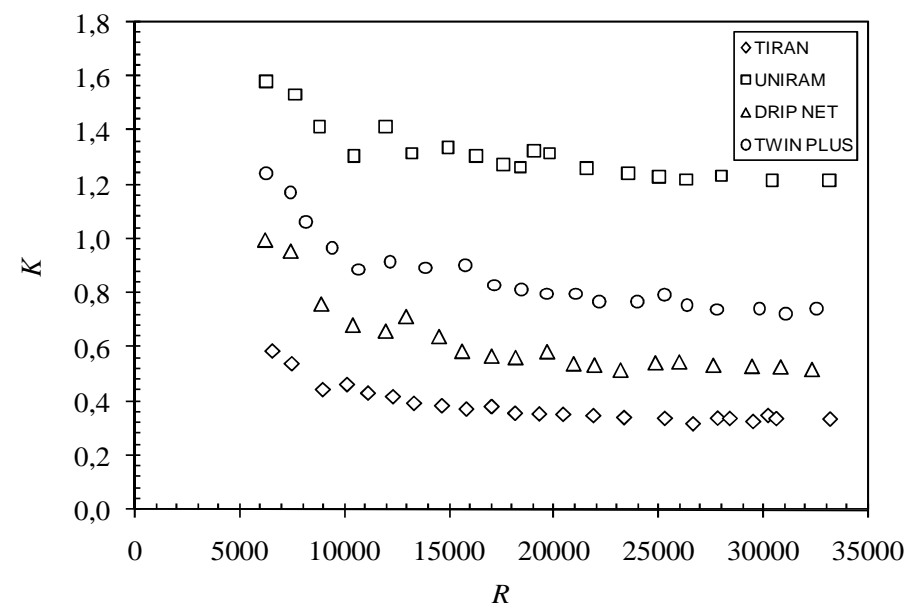

FIGURA 4. Valores médios observados de $K$ em função de $R$. Observed average values of $\boldsymbol{K}$ in relation to $R$.

PROVENZANO et al. (2005) desenvolveram uma relação que permite prever o valor de $K$ em função da razão entre o diâmetro da seção transversal do tubo $\left(D_{t}\right)$ e o diâmetro equivalente da área de escoamento no local em que o gotejador está inserido $\left(D_{r}\right)$, para gotejadores não integrados, para o domínio $1,04<D_{t} / D_{r}<1,16\left(1,08<A_{t} / A_{r}<1,35\right)$. Para ampliar o domínio dessa função $(1,08<$ $\left.A_{\mathrm{t}} / A_{r}<1,75\right)$, agruparam-se os resultados deste experimento com os resultados de PROVENZANO et al. (2005) e ajustou-se nova função potencial aos pares $(K, I O)$, resultando no modelo apresentado na Figura 5, que é caracterizado por coeficiente de determinação de 93,62\% e erro-padrão da média de 0,0025. A forma matemática da equação de regressão é fisicamente coerente, pois, quando $I O$ é igual a zero ( $r=1$, nenhuma obstrução), o coeficiente $K$ é zero.

As aproximações para as perdas de carga localizadas em termos de comprimento equivalente $\left(L_{e}\right)$ possuem validades práticas (JUANA et al., 2002; REDDY, 2003), porém deve ser observado 
que $f$ varia ao longo da linha lateral com a variação da vazão. Pode, também, variar em uma lateral específica em decorrência da variação da pressão de entrada que afeta a vazão do emissor. Consequentemente, $L_{e}$ não é constante para um dado valor de $K$ nem $K$ é constante para um dado valor de $L_{e}$.

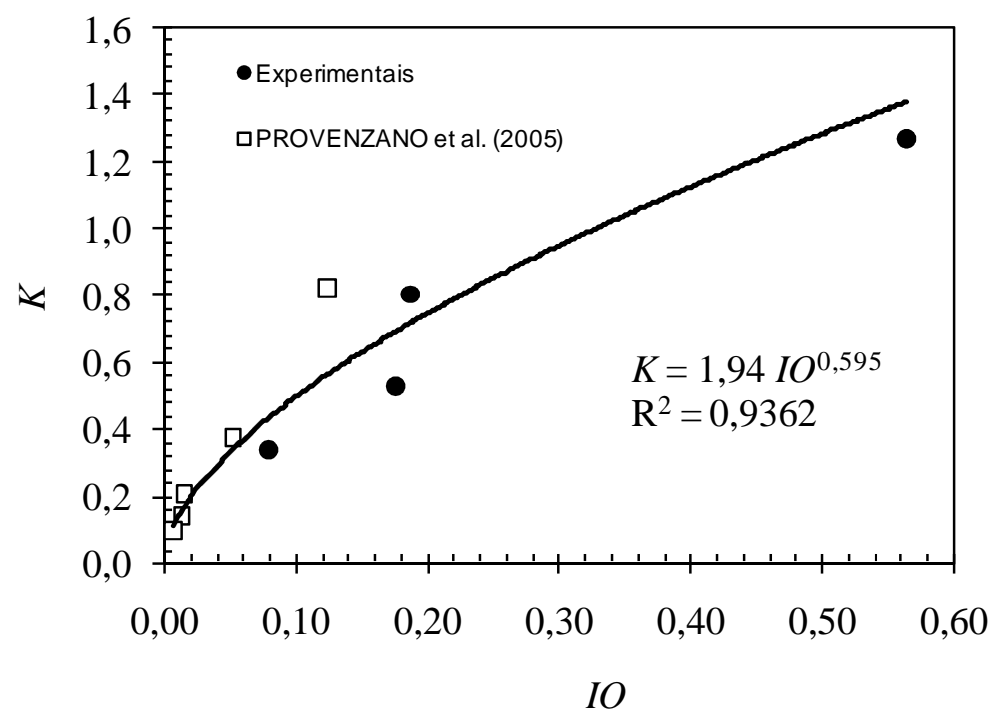

FIGURA 5. Relação entre razão de obstrução e coeficiente de carga cinética para os gotejadores analisados. Relation between obstruction ratio and kinetic load coefficient for the analyzed emitter.

Assumindo um comprimento equivalente constante para cada gotejador igualmente espaçado, para fins práticos, pode-se expressar $L_{e}(\mathrm{~m})$ em função de $h_{f e}(\mathrm{~m}), D(\mathrm{~m})$ e $Q\left(\mathrm{~m}^{3} \mathrm{~s}^{-1}\right)$. Simplificando a eq.(6) e substituindo $c$ por 0,296, tem-se a [eq.(10)]:

$$
\mathrm{L}_{\mathrm{e}}=43,43264 \mathrm{~h}_{\mathrm{fe}} \mathrm{Q}^{-1,75} \mathrm{D}^{4,75} \mathrm{v}^{-0,25}
$$

em que,

$h_{f e}$ pode ser calculado pela eq.(7) com os coeficientes $k$ e $\alpha$ obtidos na Tabela 3 para cada modelo de gotejador ou em função do índice de obstrução (Figura 5).

O cálculo da perda de carga total na linha lateral, considerando o comprimento equivalente para expressar a perda de carga localizada $\left(h_{f T}\right)$, pode ser obtido multiplicando-se o comprimento da tubulação $(L)$ por um fator $\lambda$ [eq.(11)], tal que:

$$
\lambda=\left(1+\frac{\mathrm{L}_{\mathrm{e}}}{\mathrm{S}_{\mathrm{e}}}\right)
$$

sendo $S_{e}$ o espaçamento entre gotejadores (m).

Assumindo a validade prática da perda de carga localizada expressa em comprimento equivalente, a perda de carga total na linha de gotejadores pode ser calculada em função da vazão de entrada $\left(Q, \mathrm{em} \mathrm{m}^{3} \mathrm{~s}^{-1}\right)$, do diâmetro interno da tubulação $(D$, em $\mathrm{m})$, do comprimento da tubulação $(L$, em $\mathrm{m})$, do espaçamento entre gotejadores $\left(S_{e}\right.$, em $\left.\mathrm{m}\right)$, do comprimento equivalente $\left(L_{e}\right.$, em $\left.\mathrm{m}\right)$, da viscosidade cinemática da água $\left(v\right.$, em $\left.\mathrm{m}^{2} \mathrm{~s}^{-1}\right)$ e do fator de redução de perda de carga $(F)$ para tubulações com múltiplas saídas [eq.(12)]:

$$
\mathrm{h}_{\mathrm{fT}^{\prime}}=2,301810^{-2} \mathrm{Q}^{1,75} v^{0,25} \mathrm{D}^{-4,75} \mathrm{~L} \lambda \mathrm{F}
$$


$\mathrm{Na}$ Tabela 4, apresentam-se os resultados de aplicações das equações de perda de carga desenvolvidas para os tubos gotejadores estudados. Dimensionou-se uma linha lateral em nível, por um procedimento passo a passo (PROVENZANO \& PUMO, 2004), iniciando-se pelo último gotejador da linha e considerando que o primeiro gotejador estava situado a $1 \mathrm{~m}$ do início da lateral. Para os gotejadores autocompensantes, assumiu-se a pressão no final da linha $\left(H_{\text {fin }}\right)$ igual a $10 \mathrm{~m}$, a pressão no início $\left(H_{\text {in }}\right)$ no máximo igual a $25 \mathrm{~m}$ e a vazão média dos gotejadores igual à declarada para cada modelo. A perda de carga localizada foi calculada para cada modelo de gotejador, utilizando-se a eq.(7) com os coeficientes da Tabela (3).

TABELA 4. Comparação entre perda de carga estimada pela eq. (12) e perda de carga estimada pelo dimensionamento passo a passo de uma linha lateral para os tubos gotejadores estudados. Comparison between estimated head loss by eq.(12) and estimated head loss calculated by step-by-step for lateral lengths for emitters.

\begin{tabular}{lcccc}
\hline \multirow{2}{*}{ Parâmetros } & \multicolumn{4}{c}{ Modelo de Tubo Gotejador } \\
\cline { 2 - 5 } & Twin Plus & Drip Net & Uniram & Tiran \\
\hline Razão de obstrução $\left(\mathrm{A}_{\mathrm{r}} / \mathrm{A}_{\mathrm{t}}\right)$ & 0,697 & 0,704 & 0,571 & 0,780 \\
$\mathrm{q}_{\mathrm{m}}\left(\mathrm{L} \mathrm{h}^{-1}\right)$ & 1,80 & 1,60 & 2,30 & 1,95 \\
$\mathrm{D}(\mathrm{mm})$ & 15,5 & 15,2 & 14,4 & 14,4 \\
$\mathrm{~S}_{\mathrm{e}}(\mathrm{m})$ & 1,00 & 0,75 & 0,75 & 0,70 \\
$\mathrm{~N}$ & 350 & 405 & 260 & 163 \\
$\mathrm{~L}(\mathrm{~m})$ & 350,0 & 304,0 & 196,0 & 114,4 \\
$\mathrm{Q}_{\text {in }}\left(\mathrm{L} \mathrm{h}^{-1}\right)$ & 630,0 & 648,0 & 600,3 & 318,3 \\
$\mathrm{H}_{\text {in }}(\mathrm{m})$ & 24,98 & 24,96 & 24,93 & 11,00 \\
$\mathrm{H}_{\text {fin }}(\mathrm{m})$ & 10,00 & 10,00 & 10,00 & 9,00 \\
$\mathrm{H}_{\mathrm{m}}(\mathrm{m})$ & 14,13 & 14,10 & 14,08 & 9,60 \\
$\mathrm{H}_{\text {var }}(\mathrm{m})$ & 59,97 & 59,94 & 59,90 & 18,18 \\
$\mathrm{~h}_{\mathrm{f}}(\mathrm{m})$ & 10,04 & 10,02 & 7,34 & 1,51 \\
$\mathrm{~h}_{\mathrm{fe}}(\mathrm{m})$ & 4,94 & 4,93 & 7,58 & 0,49 \\
$\mathrm{~h}_{\mathrm{fT}}(\mathrm{m})$ & 14,98 & 14,95 & 14,92 & 2,00 \\
$\left(\mathrm{~h}_{\mathrm{fe}} / \mathrm{h}_{\mathrm{fT}}\right) 100$ & 32,98 & 32,98 & 50,80 & 24,50 \\
$\mathrm{~h}_{\mathrm{fT}}(\mathrm{m})$ & 14,73 & 14,73 & 14,82 & 1,89 \\
$\Delta \mathrm{h}_{\mathrm{f}}(\%)$ & 1,67 & 1,47 & 0,67 & 5,50 \\
\hline
\end{tabular}

Para o gotejador não autocompensante, com relação à vazão-pressão $q=0,659 H^{0,481}$ ( $q$ em $\mathrm{Lh}^{-1}$ e $H$ em m), considerou-se a variação máxima de pressão na linha igual a $20 \%\left(H_{v a r}=\left(H_{\max }-\right.\right.$ $\left.H_{\min }\right) / H_{\max }=0,20$ ) e a pressão disponível no início da linha igual a $11 \mathrm{~m}$. Para estimar o número de Reynolds, utilizou-se $v=1,01 \times 10^{-6} \mathrm{~m}^{2} \mathrm{~s}^{-1}$ (temperatura de $20{ }^{\circ} \mathrm{C}$ ), e para o regime de transição, utilizou-se a aproximação SILVERBERG \& MANADILI (1997). O fator de redução de perda de carga para tubos com múltiplas saídas $(F)$ foi calculado pela aproximação proposta por SCALOPPI (1988).

Observa-se, na Tabela 4, que a perda de carga localizada $\left(h_{f e}\right)$ representa um valor expressivo na linha lateral, sendo que o gotejador Tiran apresentou a menor percentagem em relação à perda de carga total $\left(\left(h_{f e} / h_{f T}\right) 100=24,50 \%\right)$, e o gotejador Uniram, a maior $(50,80 \%)$. Pode-se também observar a utilidade prática do conceito de comprimento equivalente para considerar a perda de carga localizada na lateral [eq.(12)]. Em relação ao método de dimensionamento passo a passo, a utilização da eq.(12) resultou em diferenças de perda de carga total $\left(\Delta h_{f}\right)$ inferiores a 1,7\% para os gotejadores autocompensantes, enquanto, para o gotejador Tiran, essa diferença foi maior $(5,5 \%)$, porém limitante para projeto de linhas laterais de sistemas de irrigação por gotejamento. 


\section{CONCLUSÕES}

Para cada modelo tubo-gotejador, o coeficiente de carga cinética $(K)$ foi praticamente independente do número de Reynolds para $R>10.000$, sugerindo que cada sistema pode ser caracterizado por um valor médio de $K$ em função do índice de obstrução caracterizado pela razão de obstrução. Observou-se considerável variabilidade dos valores de $K$ entre os sistemas investigados.

Os resultados encontrados indicaram que o uso de comprimento equivalente $\left(L_{e}\right)$ constante para estimar a perda de carga localizada em uma linha lateral é um critério válido e prático. A maior diferença percentual encontrada entre a perda de carga total calculada pelo método iterativo passo a passo e pelo uso de $L_{e}$ constante, calculado com a vazão de entrada na lateral, foi 5,5\% para o gotejador não autocompensante. Para os gotejadores autocompensantes, as diferenças foram inferiores a $1,7 \%$.

\section{AGRADECIMENTOS}

Ao Ministério da Ciência e Tecnologia (MCT), ao Conselho Nacional de Desenvolvimento Científico e Tecnológico (CNPq) e à Fundação de Amparo à Pesquisa do Estado de São Paulo (FAPESP), pelo apoio financeiro a esta pesquisa, por meio do Instituto Nacional de Ciência e Tecnologia em Engenharia da Irrigação (INCTEI).

\section{REFERÊNCIAS}

AL-AMOUD, A.I. Significance of energy losses due to emitter connections in trickle irrigation lines. Jounal of Agriculture Engineering Research, Silsoe, v.60, n1, p.1-5, 1995.

ALVES, P.R.V.; PORTO, R. Coeficiente geométrico para estimativa da perda de carga localizada em linhas laterais de irrigação por gotejamento. Engenharia Agrícola, Jaboticabal, v.22, n.1, p.5159, 2002.

BAGARELO, V.; FERRO, V.; PROVENZANO, G.; PUMO, D. Experimental study on flow resistance law for small-diameter plastic tipes. Journal of Irrigation and Drainage Engineering, New York, v.121, n.5, p.313-316, 1995.

BAGARELO, V.; FERRO, V.; PROVENZANO, G.; PUMO, D . Evaluating pressure losses in dripirrigation lines. Journal of Irrigation and Drainage Engineering, New York, v.123, n.1, p.1-7, 1997.

BERNUTH, R.D. Von. Simple and accurate friction loss equation for plastic pipe. Journal of Irrigation and Drainage Engineering, New York, v.116, n.2, p.294-298, 1990.

BERNUTH, R.D. Von; WILSON, T. Friction factor for small diameter plastic pipes. Journal of Hydraulics Engineering, New York, v.115, n.2, p.183-192, 1989.

HOWELL, T.A.; BARINAS, F.A. Pressure losses across trickle irrigation fittings and emitters. Transactions of the American Society of Agricultural Engineers, St. Joseph, v.23, n.4, p.928-933, 1980.

JUANA, L.; RODRIGUES-SINOBAS, L.; LOSADA, A. Determining minor head losses in drip irrigation laterals. I: Methodology. Journal of Irrigation and Drainage Engineering, New York, v128, n.6, p.376-384, 2002.

PROVENZANO, G.; PUMO D. Experimental analysis of local pressure losses for microirrigation laterals. Journal of Irrigation and Drainage Engineering, New York, v.130, n.4, p.318-324, 2004.

PROVENZANO, G.; PUMO, D.; DI DIO, P. Simplified procedure to evaluate head losses in drip irrigation lateral. Journal of Irrigation and Drainage Engineering, New York, v.131, n.6, p.525$532,2005$. 
REDDY, K.Y. Evaluation of on-line trickle irrigation emitter barb losses. Journal of the Institution of Engineers- $A G$, Kharagpur, v.84, n.1, p.42-47, 2003.

ROMEO, E.; ROYO, C.; MONZÓN, A. Improved explicit equations for estimation of the friction factor in rough and smooth pipes. Chemical Engineering Journal, Amsterdam, v.86, n.3, p.369-374, 2002.

SCALOPPI, E.J. Adjusted F factor for multiple-outlet pipes. Journal of Irrigation and Drainage Engineering, New York, v.114, n.1, p.169-174, 1988.

SILVERBERG, P.M; MANADILI, G. Replace implicit equations with signomial functions: A simple equation makes a user-friendly fit of friction factor chart. Chemical Engineering, New York, v.104, n.8, p.129, 1997.

VILELA, L.A.A.; SOCCOL, O.J.; GERVÁZIO, E.S.; FRIZZONE, J.A.; BOTREL, T.A. Alteração no diâmetro de tubos de polietileno submetidos a diferentes pressões. Revista Brasileira de Engenharia Agrícola e Ambiental, Campina Grande, v.7, n.1, p.182-185, 2003.

YOO, D.H.; SINGH, V.P. Two methods for the computation of commercial pipe friction factors. Journal of Hydraulic Engineering, New York, v.131, n.8, p.694-704, 2005. 